\title{
Bayesian model of Snellen visual acuity
}

\author{
Oscar Nestares and Rafael Navarro \\ Instituto de Óptica “Daza de Valdés,” Consejo Superior de Investigaciones Científicas, Serrano 121, \\ 28006 Madrid, Spain
}

\author{
Beatriz Antona \\ Departamento de Optica, Universidad Complutense de Madrid, Arcos de Jalón s/n, 28037 Madrid, Spain
}

Received June 20, 2002; revised manuscript received January 6, 2003; accepted January 7, 2003

\begin{abstract}
A Bayesian model of Snellen visual acuity (VA) has been developed that, as far as we know, is the first one that includes the three main stages of VA: (1) optical degradations, (2) neural image representation and contrast thresholding, and (3) character recognition. The retinal image of a Snellen test chart is obtained from experimental wave-aberration data. Then a subband image decomposition with a set of visual channels tuned to different spatial frequencies and orientations is applied to the retinal image, as in standard computational models of early cortical image representation. A neural threshold is applied to the contrast responses to include the effect of the neural contrast sensitivity. The resulting image representation is the base of a Bayesian pattern-recognition method robust to the presence of optical aberrations. The model is applied to images containing sets of letter optotypes at different scales, and the number of correct answers is obtained at each scale; the final output is the decimal Snellen VA. The model has no free parameters to adjust. The main input data are the eye's optical aberrations, and standard values are used for all other parameters, including the Stiles-Crawford effect, visual channels, and neural contrast threshold, when no subject specific values are available. When aberrations are large, Snellen VA involving pattern recognition differs from grating acuity, which is based on a simpler detection (or orientation-discrimination) task and hence is basically unaffected by phase distortions introduced by the optical transfer function. A preliminary test of the model in one subject produced close agreement between actual measurements and predicted VA values. Two examples are also included: (1) application of the method to the prediction of the VA in refractive-surgery patients and (2) simulation of the VA attainable by correcting ocular aberrations. (c) 2003 Optical Society of America

OCIS codes: $330.1070,330.4060,330.5000,330.5370,330.6110$.
\end{abstract}

\section{INTRODUCTION}

Visual acuity (VA) is the most widely used parameter to characterize the global quality of vision of a subject. Obtaining the VA is simple and it is extremely helpful in detecting the presence of visual problems, especially in performing subjective refraction. Snellen VA is obtained by asking the subject to perform a pattern-recognition task, typically under maximum-contrast conditions. The minimum size of the targets (optotypes) for which the number of correct answers is above threshold determines the value of VA.

One of the reasons for the great success of Snellen VA among clinicians is its high sensitivity to optical imperfections, such as defocus. Indeed, VA can easily detect defocus values equal to or less than $\frac{1}{4}$ diopter (D). This method, based on optotype recognition, is preferred over grating acuity or similar procedures based on a much simpler detection task. The latter procedures are hardly ever used in clinics, probably because grating acuity is less sensitive to optical ametropias, since it is affected only by the modulus of the optical transfer function (OTF), which produces a contrast loss of the grating. In contrast, more-complex patterns such as letter optotypes are also affected by the phase of the OTF. Phase distortions induced by the OTF cause artifacts such as contrast reversals ${ }^{1}$ and multiple images, which, added to the contrast losses, quickly impair the recognition task.

There has traditionally been much interest in studying the effect of refractive errors on VA. ${ }^{2,3}$ Lately, because of the rapid development of refractive and cataract surgery, this interest has increased and has extended to other visual performance metrics such as the contrast sensitivity function (CSF) and to the study of effects of other optical defects and aberrations. These techniques induce important modifications to the optics of the eye, and therefore it is critically important to be able to predict the effect of such modifications on visual performance. The widespread application of these surgery techniques is making patent the need for an accurate description and measure of the refractive properties of the eye, as is demonstrated by the increased interest in ocular aberrometry. ${ }^{4-8}$ There have been many reports of empirical data on the outcome of different surgery techniques, including optical $^{9,10}$ and visual ${ }^{11-13}$ performance, but there is still a considerable lack of realistic models that are able to predict the visual performance under different conditions, to establish realistic tolerances of optical aberrations, ${ }^{14}$ or customize the surgery ${ }^{15}$ for an optimal visual response. Ideally, realistic models must separate optical from neural contributions to the visual response. This separation makes possible a more direct testing of the impact of refractive surgery (such as PRK and LASIK). Since this kind of surgery acts on the external surface of the eye, it seems reasonable to assume that, at least for the clinically successful cases, it only modifies the optics of the eye, without affecting retinocortical structures. Under 
this assumption, changes in visual performance (VA, CSF, etc.) must be due to the optical changes induced by surgery.

Work has been done previously on modeling grating acuity from schematic eye models ${ }^{16}$ and more recently on optical modeling to calculate the appearance of the retinal image of sets of Snellen optotypes from ocular aberration data. ${ }^{17}$ However, as far as we know, the crucial patternrecognition stage has not been modeled yet. Here we propose a complete model of Snellen visual acuity that includes both optical and cortical image representation models, followed by a Bayesian pattern-recognition scheme, similar to the classical ideal-observer model. ${ }^{18}$ The optical model is characterized by the pupil function, given by its modulus (effective pupil transmission) and its phase (wave aberration). ${ }^{19}$ We consider cases of both monochromatic and polychromatic illumination. The cortical image representation is characterized by a set of visual channels ${ }^{20,21}$ plus a neural contrast threshold. ${ }^{22,23}$ It is worth noting that the set of visual channels is a highly convenient representation that makes it possible to introduce additional constraints and simplifications to the Bayesian solution on the pattern-recognition stage. Our Bayesian approach introduces robustness against the presence of optical aberrations, such as those present in human eyes. ${ }^{24}$ In fact, although there is a rather large literature on powerful pattern-recognition algorithms, these algorithms barely accommodate defocus and optical aberrations, which are prominent in the visual system. Previous research suggested that it is possible to perform pattern recognition in the presence of defocus by using a subband decomposition based on the visual channels. ${ }^{25}$

We believe that the proposed model is a powerful tool to analyze and predict the effect of optical factors (defocus, aberrations, pupil size, etc.) on visual acuity. If we know the wave-aberration function provided by optical aberrometry methods, it is possible to predict VA for different defocus, pupil sizes, or even varying aberrations for subjects who have normal neural response. The model was tested by using aberrometry data from one eye, and close agreement was obtained between predicted VA values and actual measurements of VA obtained in the clinic. In Section 2 we present the Bayesian formulation of the pattern-recognition problem in the presence of optical aberrations, as well as the optical and neural models. In Section 3 we describe how to implement these models to obtain the values of VA from the input data (aberrations, pupil size, etc.), and Sections 4 and 5 contain the results and discussion, respectively.

\section{THE MODEL}

Figure 1 shows a block diagram with the main stages and components of the model. The image of an input Snellen chart is filtered with the OTF of the optics of the eye to produce the degraded retinal image. ${ }^{26}$ The OTF depends on the pupil size, aberrations, and the Styles-Crawford apodizing effect. Retinal sampling is modeled by introducing spectral replicas of the retinal image at proper locations. Then the retinal image is processed through the early visual stages to produce a cortical image representation. Here we apply a simple schematic model, consist-

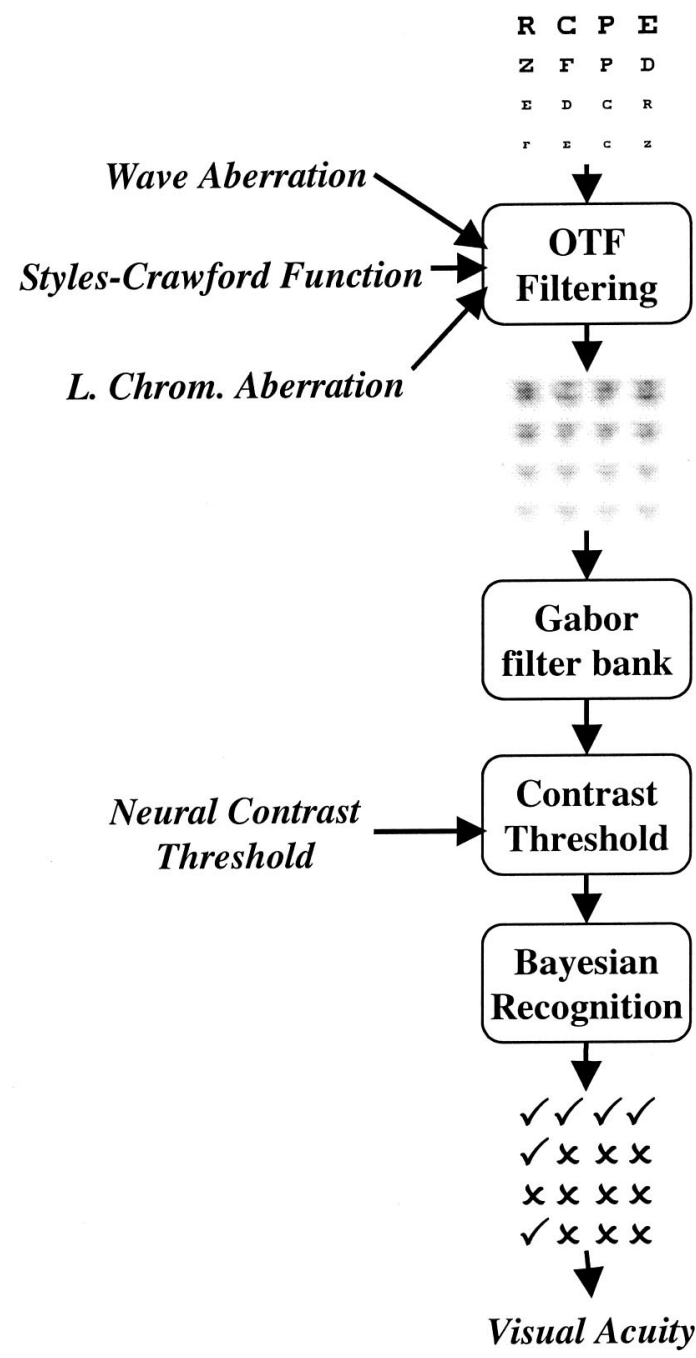

Fig. 1. Schematic diagram of the model. The input data of the optical model are ocular aberrations and Stiles-Crawford-effect parameters and a computer-generated set of optotypes at different scales corresponding to different VA values. The cortical image representation is based on a set of Gabor filters and on the neural contrast threshold. Then the Bayesian pattern recognition process is applied to the different optotypes sets, and the returned VA is the one for which the number of correct answers is above threshold.

ing of a linear filtering with a multiscale/multiorientation bank of Gabor filters to produce a set of visual channels selective to frequency and orientation, followed by an intensity normalization to obtain local contrast responses. ${ }^{27}$ A neural contrast threshold, given by the inverse of the neural contrast sensitivity, or neural transfer function (NTF) is applied, suppressing all the contrast responses below it. The resulting cortical image representation of the optically degraded object is the input to the Bayesian pattern-recognition stage. Visual acuity is obtained by applying the model to sets of Snellen optotypes and analyzing the number of correct answers for several scales.

\section{A. Optical Model}

The optical model is based on well-known standard methods. ${ }^{26,28}$ For monochromatic illumination, the optical performance of the eye is characterized by the pupil function $P$, which is a complex function describing the 
wave front at the exit pupil plane. If the incident beam is homogeneous, the amplitude at the pupil is given by the effective pupil transmission $T$, and the shape of the wave front is usually given by the wave aberration $W$, which is the difference between the actual wave front and the ideal reference sphere:

$$
P(\xi, \eta)=T(\xi, \eta) \exp \left[-i 2 \pi \frac{n}{\lambda} W(\xi, \eta)\right],
$$

where $(\xi, \eta)$ are Cartesian pupil coordinates, usually given in millimeters; $\lambda$ is the wavelength of the light; and $n$ is the refraction index of the vitreous body. The measured wave aberration $W$ is described in terms of a Zernike polynomial $\left(z_{k}\right)$ expansion, according to the Optical Society of America's standard. ${ }^{29}$ Each Zernike coefficient $c_{k}$ represents the amount of a particular aberration mode (prism, defocus, astigmatism, coma, spherical aberration, etc). In what follows we use a seventh-order expansion, so that $W$ is given by a set of 35 Zernike coefficients (the zeroorder piston term is ignored):

$$
W(\xi, \eta) \approx \sum_{k=1}^{35} c_{k} z_{k}\left(\xi / R_{p}, \eta / R_{p}\right) .
$$

Zernike polynomials form a complete basis on a circle of unit radius. Thus they are functions of pupil coordinates normalized by the pupil radius $R_{p}$ for which the wave aberration was measured. The transmission function $T$ is modeled with use of a Gaussian apodization function given by the Stiles-Crawford effect (SCE). When no data are available for a specific subject, we use a standard average SCE function. ${ }^{30}$ Although the optical model will be more accurate if specific patient data are available, in most cases the standard SCE is a good enough approximation. The standard SCE considers that the peak sensitivity is at the pupil center, so that pupil transmittance $T$ will be given by the standard SCE expression within the pupil circle and be zero outside:

$$
T(\xi, \eta)=\exp \left[-\frac{\rho}{2}\left(\xi^{2}+\eta^{2}\right)\right] \operatorname{circ}\left(\sqrt{\xi^{2}+\eta^{2}}, R\right),
$$

where $R$ is the pupil radius for which we are computing $T$, which must be less than or equal to $R_{p} ; \operatorname{circ}(r, R)$ is a circle function, equal to 1 for $r<R$ and 0 elsewhere; and $\rho=0.12$ is the standard SCE parameter. Once the pupil function $P$ is fully specified, we can compute the $\mathrm{OTF}^{31}$ for the desired pupil diameter $R$, as the autocorrelation of the pupil function $P$ :

$$
\operatorname{OTF}(u, v)=P(\xi / \lambda, \eta / \lambda) \otimes P(\xi / \lambda, \eta / \lambda),
$$

where $(u, v)$ are dimensionless $\left(\operatorname{cycles}^{-1}\right)$ spatial frequencies. For the experimental data used here the wavelength of the light is $\lambda=543 \mathrm{~nm} .7,10$

The last step is to obtain the optical retinal image of the extended input object. In what follows, the object will be a Snellen chart containing one or more optotypes at different scales. The retinal image $I\left(x^{\prime}, y^{\prime}\right)$ of an extended object $O(x, y)$, is given by $^{26}$ :

$$
I\left(x^{\prime}, y^{\prime}\right)=\mathrm{FT}^{-1}[\mathrm{FT}[O(x, y)] \operatorname{OTF}(u, v)],
$$

where FT means Fourier transform.
So far we have described the monochromatic optical model. However, Snellen charts are typically projected with use of polychromatic, white light. The polychromatic version must take into account chromatic aberrations and the specific weighting of each wavelength given by the illuminant spectral energy distribution and by the subject spectral sensitivity curve. Illumination conditions in VA measurements can change from clinic to clinic. For this reason, here we use the equienergetic spectral distribution as our standard, which means constant weight for all wavelengths. To characterize the subject's response, we use the weights given by the CIE standard observer photopic spectral sensitivity $V_{\lambda} \cdot{ }^{32}$ An accurate model of the chromatic aberrations requires measuring the wave aberration for several wavelengths, sampling finely the visible spectrum (up to 40 wavelengths have been used previously ${ }^{33}$ ). However, aberration data are usually available for one or two wavelengths, and moreover, objective aberrometers cannot measure the transverse chromatic aberration ${ }^{34}$ (TCA). Therefore here again we use a simple model considering only the longitudinal chromatic aberration (LCA), which is relatively constant among subjects. ${ }^{35}$ LCA is a wavelengthdependent defocus, which is easily introduced by modifying the defocus Zernike coefficient $\left(Z_{d}\right)$, according to the following expression:

$$
Z_{d}=\frac{D R^{2}}{4}
$$

where $D$ is the value of defocus in diopters. Based on clinicians' experience that defocus values of less than $\frac{1}{4} \mathrm{D}$ do not make much difference in terms of VA, we have sampled the visible spectrum in $\frac{1}{4}$-D steps of LCA. Because for LCA of $\frac{1}{2} \mathrm{D}$ the weighting factor $V_{\lambda}$ decays approximately one order of magnitude or even more (for the long wavelengths), we consider only three wavelengths for LCA, those corresponding to a defocus of $-\frac{1}{4}, 0$, and $\frac{1}{4}$ D. Finally, the polychromatic retinal image is obtained as the weighted average of the monochromatic retinal images for the three wavelengths considered or, equivalently, by applying the polychromatic OTF, also obtained as a weighted average of monochromatic OTFs.

\section{B. Retinal Sampling}

Once the image is formed onto the retina, it is sampled by the photoreceptor array. We have simulated the spectral aliasing caused by this sampling by replicating the spectrum of the retinal image at the six vertices of a hexagon inscribed in a circle of radius equal to 120 cycles/deg. This is an approximation of the retinal sampling at the center of the fovea, where the cones are packed in an approximate hexagonal way, the distance between them corresponds to a sampling frequency of $\sim 120$ cycles/deg. This effect is important when the optical degradation is not severe and/or for small scales of the optotypes. Usually neither condition is present when there are optical aberrations. However, we found this step very important when testing the model by using an aberration-free eye, where the only optical blur is caused by diffraction and typically attainable VA are high, requiring small scale of the optotypes. 


\section{Cortical Contrast Representation}

The retinal image is encoded by using a simplified schematic model of early visual processing based on applying a bank of filters tuned at different scales and orientations, and an intensity normalization to obtain responses in contrast units. ${ }^{27}$ Among the different filters proposed to model the receptive-field weighting functions of cells in area V1, we chose Gabor functions, although other choices (such as Gaussian derivatives) will not significantly change the model output. The parameters of the Gabor filter bank were chosen according to biological plausibility and computational efficiency ${ }^{36}$ :

- Radial bandwidth of 1 octave.

- Form factor of 1 (isotropic Gaussian envelope).

- Three scales distributed in octaves.

- Highest radial tuning frequency of 32 cycles/deg.

This value is given by $\sim \frac{1}{4}$ of the average human cone spatial frequency at the center of the fovea. ${ }^{37}$ Again, this standard value can be easily adapted to the subject, provided that specific experimental data are available.

- Four orientations (0, 45, 90, and $135 \mathrm{deg})$.

With these parameters it is possible to implement the filter bank efficiently in the spatial domain by using separable convolutions and a pyramidal strategy to obtain the coarser scales. ${ }^{36}$ An interesting property of the multichannel representation is that it makes it possible to compute contrast responses by normalizing the filter responses by a low-pass-filtered version of the input image. ${ }^{27}$ Figure 2 plots radial profiles of the frequency response of the Gabor filters for the three scales considered, together with a standard $\mathrm{NTF}^{23}$ whose inverse gives the threshold contrast for each spatial frequency, as explained below. Lower frequencies were not considered with the choice of three scales, mainly for computational efficiency, because considering them would force us to start from higher-resolution images. Besides, these frequencies are not crucial for the VA task.

\section{Neural Contrast Threshold}

Next, the model considers the limited neural performance in terms of the neural contrast threshold, often given by its inverse, the neural contrast sensitivity or the NTF by some authors. The neural contrast sensitivity can be

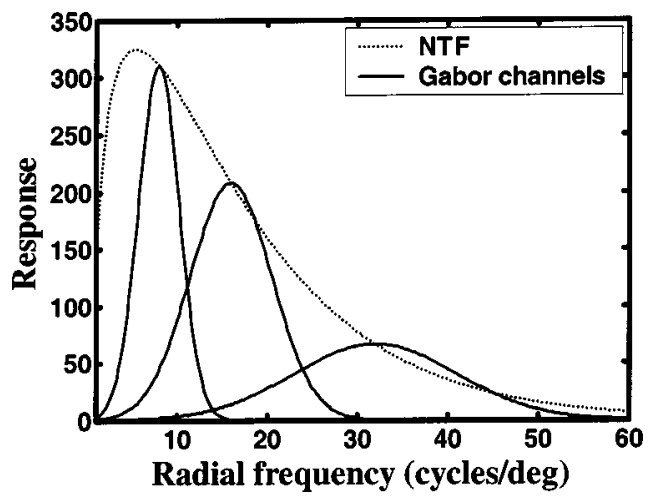

Fig. 2. Plot of the NTF (inverse of neural contrast threshold) and the three frequency Gabor channels considered in the current version of the model. (Each frequency channel contains four orientation channels). measured either directly, bypassing the optics of the eye by interferometric techniques, ${ }^{22,38}$ or indirectly, discounting numerically the effect of the optics dividing the CSF by the optical modulation transfer function ${ }^{23}$ (MTF). If the subject's neural contrast sensitivity is not available, we use an average standard NTF taken from Ref. 23, which is displayed in Fig. 2. Using this standard NTF is not advised when the subject presents visual deficits known to specifically affect the NTF (e.g., amblyopia). In this case, it would be necessary to measure the NTF of the subject to obtain accurate VA predictions.

The neural contrast threshold, given by the inverse of the NTF, is applied to the contrast responses of the different Gabor channels. For each channel we apply a single threshold value, given by the inverse of the NTF value at the central tuning frequency of the channel. This is illustrated in Fig. 2, where we have plotted the radial frequency response of the Gabor channels for the three scales considered, each channel scaled by the NTF value at its central tuning frequency.

\section{E. Bayesian Recognition of Degraded Images of Optotypes}

The last stage of the model is the recognition of the optically degraded optotypes. Traditional methods for pattern recognition (such as correlation-based methods) fail to recognize the targets even in the presence of moderate or even small optical degradations, such as defocus. Hence, when we attempted standard pattern-recognition methods, we obtained heavily underestimated predictions of VA. A previous study ${ }^{25}$ demonstrated that a subband decomposition of the recognition filter makes it possible to develop pattern-recognition methods robust to defocus by strongly decreasing the probability of false alarms (wrong answers). Here we further develop and generalize that idea within a Bayesian framework.

The Bayesian recognition stage requires that the observed degraded letter optotype be compared with all the letters in the alphabet. Here we assume that the subject had previously learned the shapes of the letters of the alphabet under optimal conditions, i.e., at a scale and contrast well above threshold. Even though the retinal images of the letters are always affected by blur, most observers have the opportunity to learn the alphabet with letter scales where the distortions caused by blur are negligible. We also assume that the subject does not explicitly estimate the degradation introduced by his eye. Therefore in the proposed model the observed degraded letter is compared against a nondegraded version of the letter, and the degradation is implicitly estimated by the proposed Bayesian pattern-recognition method. It is true that the subject could have learned directly the degraded versions of the letters, but this is less realistic if we take into account that the degradation introduced by the optics of the eye is variable and depends not only on the aberrations of the system but also on pupil size, defocus, and other factors such as accommodation state. Furthermore, the relative effect of blur changes dramatically with letter size. Therefore this approach would require learning the shapes of the letters for different optical degradations and letter sizes, which seems less realistic than having a single internal representation of the alphabet. 
The main drawback of our assumptions is that the visual system would have to face a blur-estimation problem every time, and the success of the recognition would therefore depend on the ability of the subject to estimate the degradation introduced by the OTF. This problem has some analogies with blind deconvolution, but here the input signals are highly constrained, because they belong to a reduced, finite, and well-known alphabet, making the problem much easier for the visual system. In addition, by introducing spatial-frequency visual channels, the blur-estimation problem can be strongly simplified, yielding a simple, robust, and affordable solution that is basically consistent with current models of early visual processing. This is the key feature of this approach; that is, frequency and orientation channels are essential to the VA model because they allow one to bypass an ill-posed, numerically unstable problem and finally arrive at a much simpler, easy-to-compute, and stable approximate solution. Nevertheless, in Section 5 we will compare this model with a more standard ideal-observer model, ${ }^{18}$ in which it is assumed that the blur is known and the observed optically degraded letters, contaminated with additive noise, are compared against an optically degraded, noise-free alphabet.

Even taking into account the prior information provided by the well-known alphabet, the general recognition problem is ill-posed, and we need to rely on further additional constraints. Here we use the model of early visual processing described above, which allows us to make several reasonable assumptions, helping us to greatly simplify the Bayesian formulation of the OTF estimation problem. Following that model, the degraded retinal image is decomposed in subbands by means of a Gabor filter bank. Here we introduce the strongest and most simplifying assumption: that the OTF is constant inside each Gabor channel. Since the OTF is a complex filter in the frequency domain, this means that within a Gabor channel, the OTF is approximated by a constant multiplicative factor (channel average MTF) and a global shift (channel average phase). The validity of this assumption will depend on the number of visual channels (more channels would provide a better sampling of the OTF) and the shape of the OTF (the more complicated the OTF, the higher the number of samples is required). Published data on the eye's MTF (Ref. 39) suggest a good general fit with a multiscale octave-based scheme, since the MTF tends to vary more steeply toward the lower frequencies and is shallower for the highest frequencies. This, however, does not hold for phase distortions, which can be important for large aberrations and/or defocus, and hence the number of channels will limit performance in the recognition task. In other words, this model will yield low VA values when phase distortions are important, but for a visual model this does not seem unrealistic since the performance of human observers is also limited by strong phase distortions. We have to keep in mind that the visual system has limited VA, and current models also assume a limited number of visual channels. The number of channels can be freely varied in the model, but here we have applied a simplicity criterion, using the minimum number of channels that can capture the essential features of this approach. The validity of this and other pos- sible choices will depend mainly on the agreement between measured data and model predictions.

With the previous assumption, we can formulate the following observation model for each of the Gabor filtered versions of the retinal image:

$$
\begin{aligned}
o_{i}(\mathbf{x}) & =[h(\mathbf{x}) * c(\mathbf{x})] * g_{i}(\mathbf{x})+\eta_{i}(\mathbf{x}) \\
& \approx h_{i} c_{i}\left(\mathbf{x}-\mathbf{u}_{i}\right)+\eta_{i}(\mathbf{x}), \quad i=1, \ldots, N_{c}
\end{aligned}
$$

where the observed $o_{i}(\mathbf{x})$ is the degraded retinal image filtered with the $i$ th Gabor filter $g_{i}(\mathbf{x})$ and contaminated with additive noise, $\eta_{i}(\mathbf{x}), h(\mathbf{x})$ is the impulse response of the optical degradation, and $c(\mathbf{x})$ is the input test image containing one optotype. The intensity normalization has been omitted for the sake of simplicity, but we have to bear in mind that the convolution with the Gabor filter is followed by that intensity normalization so that the observed magnitude is contrast. The right-hand side is obtained by applying the previous approximation, $c_{i}(\mathbf{x})$ is the input image filtered with the $i$ th Gabor channel (contrast units), and $\left(h_{i}, \mathbf{u}_{i}\right)$ are respectively the constant modulation factor and the global shift approximating the OTF within the bandwidth of the $i$ th Gabor filter. $N_{c}$ is the number of Gabor channels.

Given the previous observation model, we can formulate the joint posterior probability for the original input character $\mathbf{c}$ as well as the approximated linear degradation parameters $\left\{h_{i}, \mathbf{u}_{i}\right\}$, given the observations $\left\{\mathbf{o}_{i}\right\}$; applying the Bayes rule we obtain

$$
p\left(\mathbf{c},\left\{h_{i}, \mathbf{u}_{i}\right\} \mid\left\{\mathbf{o}_{i}\right\}\right)=K p\left(\left\{\mathbf{o}_{i}\right\} \mid \mathbf{c},\left\{h_{i}, \mathbf{u}_{i}\right\}\right) p(\mathbf{c}) p\left(\left\{h_{i}, \mathbf{u}\right\}\right),
$$

where for notational convenience we have expressed images as intensity vectors; $K$ is a normalization constant. The posterior probability equals the likelihood (or conditional probability of the observations given the input letter and the degradation parameters) multiplied by the prior probability, where we have assumed that the input character $\mathbf{c}$ is independent of the OTF model parameters $\left\{h_{i}, \mathbf{u}_{i}\right\}$. If we further assume a flat constant prior for the degradation parameters $\left\{h_{i}, \mathbf{u}_{i}\right\}$, the posterior probability is finally

$$
p\left(\mathbf{c},\left\{h_{i}, \mathbf{u}_{i}\right\} \mid\left\{\mathbf{o}_{i}\right\}\right)=K^{\prime} p\left(\left\{\mathbf{o}_{i}\right\} \mid \mathbf{c},\left\{h_{i}, \mathbf{u}_{i}\right\}\right) p(\mathbf{c}),
$$

where $K^{\prime}$ is another normalization constant. The maximum a posteriori (known as MAP) estimator for the input character $\hat{\mathbf{c}}$ and for the OTF parameters $\left\{\hat{h}_{i}, \hat{\mathbf{u}}_{i}\right\}$ is the one that maximizes the posterior probability in Eq. (9),

$$
\left(\hat{\mathbf{c}},\left\{\hat{h}_{i}, \hat{\mathbf{u}}_{i}\right\}\right)=\underset{\left(c,\left\{h_{i}, \mathbf{u}_{i}\right\}\right)}{\arg \max } p\left(\left\{\mathbf{o}_{i}\right\} \mid \mathbf{c},\left\{h_{i}, \mathbf{u}_{i}\right\}\right) p(\mathbf{c}),
$$

where the likelihood function $p\left(\left\{\mathbf{o}_{i}\right\} \mid \mathbf{c},\left\{h_{i}, \mathbf{u}_{i}\right\}\right)$ is given by the probability density function of the noise according to the observation model in relation (7). If we further assume conditional independence between channels and between spatial locations inside the channels, the likelihood is given by

$$
p\left(\left\{\mathbf{o}_{i}\right\} \mid \mathbf{c},\left\{h_{i}, \mathbf{u}_{i}\right\}\right)=\prod_{i=1}^{N_{c}} \prod_{\mathbf{x}} p_{\eta_{i}}\left(o_{i}(\mathbf{x})-h_{i} c_{i}\left(\mathbf{x}-\mathbf{u}_{i}\right)\right) .
$$


Next we incorporate the prior on the input image c, which is determined by the fact that the input character belongs to a finite alphabet in which all the characters are equiprobable. Thus this prior can be expressed as a sum of delta functions, resulting in the following posterior probability:

$$
\begin{aligned}
p\left(\mathbf{c},\left\{h_{i}, \mathbf{u}_{i}\right\} \mid\left\{\mathbf{o}_{i}\right\}\right) \propto & \prod_{i=1}^{N_{c}} \prod_{\mathbf{x}} p_{\eta_{i}}\left(o_{i}(\mathbf{x})-h_{i} c_{i}\left(\mathbf{x}-\mathbf{u}_{i}\right)\right) \\
& \times\left[\sum_{j=1}^{N} \delta\left(\mathbf{c}-\mathbf{c}^{j}\right)\right]
\end{aligned}
$$

where $\left\{\mathbf{c}^{j}\right\}_{j=1}^{N}$ are the vectorized images corresponding to the $N$ characters in the alphabet. This prior heavily constrains the space of all the possible intensity configurations of the input image, resulting in a posterior that is different from 0 only when $\mathbf{c} \in\left\{\mathbf{c}^{j}\right\}_{j=1}^{N}$, points where we can assign a value of posterior probability given by

$$
\begin{aligned}
p\left(\mathbf{c}=\mathbf{c}^{j},\left\{h_{i}, \mathbf{u}_{i}\right\} \mid\left\{\mathbf{o}_{i}\right\}\right) & \\
& \propto \prod_{i=1}^{N_{c}} \prod_{\mathbf{x}} p_{\eta_{i}}\left(o_{i}(\mathbf{x})-h_{i} c_{i}^{j}\left(\mathbf{x}-\mathbf{u}_{i}\right)\right) .
\end{aligned}
$$

Therefore the recognition of an input character consists of first choosing the degradation parameters that maximize the probability in relation (13) for each character in the alphabet and then choosing the character with the largest probability, which will give us the global maximum of the posterior probability distribution. Such maximization can be done separately for each channel, followed by multiplying the maximum probability values. For channel $i$, and assuming Gaussian white noise, the maximization of the probability is equivalent to the minimization of the following error function:

$$
E_{i}^{j}=\sum_{\mathbf{x}}\left[o_{i}(\mathbf{x})-h_{i} c_{i}^{j}\left(\mathbf{x}-\mathbf{u}_{i}\right)\right]^{2} .
$$

It can be shown (see Appendix A) that the value $\hat{\mathbf{u}}_{i}^{j}$ that minimizes this error function is the same one that maximizes the correlation function $\operatorname{corr}_{i}^{j}\left(\mathbf{u}_{i}\right)=\Sigma_{\mathbf{x}} o_{i}(\mathbf{x}) c_{i}^{j}(\mathbf{x}$ - $\left.\mathbf{u}_{i}\right)$, and that $\hat{h}_{i}^{j}=\operatorname{corr}_{i}^{j}\left(\hat{\mathbf{u}}_{i}^{j}\right) / K_{i}^{j}$, where $K_{i}^{j}$ $=\Sigma_{\mathbf{x}}\left[c_{i}^{j}(\mathbf{x})\right]^{2}$. This leads to the following maximum value of the posterior probability for character $j$ :

$$
\begin{aligned}
P_{j} & =\max \left\{p\left(\mathbf{c}=\mathbf{c}^{j}\left\{h_{i}, \mathbf{u}_{i}\right\} \mid\left\{\mathbf{o}_{i}\right\}\right)\right\} \\
& \propto \exp \left[\frac{1}{2 \sigma^{2}} \sum_{i=1}^{N_{c}} \hat{h}_{i}^{j} \operatorname{corr}_{i}^{j}\left(\hat{\mathbf{u}}_{i}^{j}\right)\right] .
\end{aligned}
$$

The correlation operators can be implemented efficiently in the Fourier domain. The output of this recognition procedure is the character $j$ with the largest $P_{j}$, which is independent of the noise variance $\sigma^{2}$.

\section{METHODS}

The proposed model has been implemented in MATLAB. Next we describe the procedure for predicting the VA with the model, which is an approximate simulation of the method used to measure VA in the clinics. To minimize computation, we considered a reduced alphabet of 18 characters, which includes all those characters that appear frequently in commercial charts (see bottom row in Fig. 3). The procedure starts with choosing four letters from the alphabet. A different set of four letters was chosen randomly for each value of VA, and the same set is used in all the experiments to facilitate further comparisons between the predicted values under different conditions. The letters are then resized to a scale corresponding to a preset initial decimal VA value, typically $\mathrm{VA}_{0}$ $=0.8$, so that the angle subtended by them is equal to 5 arc min divided by the decimal VA. If the number of correct answers is above a given threshold (in the following examples we permit one error), that value of VA is considered to be surpassed, and we increase the VA in 1/10 steps until the number of correct answers is less than three. If the initial number of answers does not reach the threshold, then the VA is decreased in 1/10 steps until the number of correct answers is above threshold. In both cases, the VA is the last value for which we obtained at least three of four correct answers. Figure 3 illustrates the basic procedure, although this is a simplified example showing four lines in 0.2 steps of VA.

The current version of the model is monocular. It was implemented and tested by using standard data for the SCE, longitudinal chromatic aberration, and neural contrast threshold, with actual wave-aberration data for different subjects taken from two previous studies. 7,10 These data are used here to illustrate the outcome of the model and to show its potential applications. A deeper study of these applications is beyond the scope of this paper, and therefore we cannot draw strong conclusions from the few examples presented here.

The first example is a preliminary experimental test of the model, where we compared the VA predictions of the model with actual VA measurements for one eye. We measured the VA for a normal experienced subject, RN, using standard procedures in an optometric clinic. Optotypes were projected on a screen placed $6 \mathrm{~m}$ away from the subject. Room lights were kept off to avoid contrast losses of the optotypes. Pupil size was measured three times during the procedure giving a consistent size of 5.5 $\mathrm{mm}$. Visual acuity was measured under different defocus values by introducing trial lenses. The nominal refraction for this subject is $-0.75 \mathrm{D}$ sphere and $-0.75 \mathrm{D}$ of astigmatism (the latter was left uncorrected). We were interested in scanning a $2 \mathrm{D}$ range of defocus, so the session started from the more myopic refractive state, adding +1.5 by using a trial lens. Then we took VA measurements, changing defocus in steps of $0.5 \mathrm{D}$, except that we took finer steps around the nominal refraction. We did not continue to measure VA for hyperopic refractive states, to avoid compensation of refractive error by accommodation, which seems to occur even when a $0.25 \mathrm{D}$ hyperopia is introduced (see Fig. 4 below). To prevent the subject from learning the optotypes' sequence, a single run was performed first, starting from the most myopic refractive state (lower VA); and for each refractive state, we started with the largest optotypes (lower VA). The VA was measured in four runs. The last two runs showed an improvement in VA for the intermediate refractive states, which correspond to VA values most often used in the session. This suggests a spurious learning effect; conse- 


\section{Optotype chart}

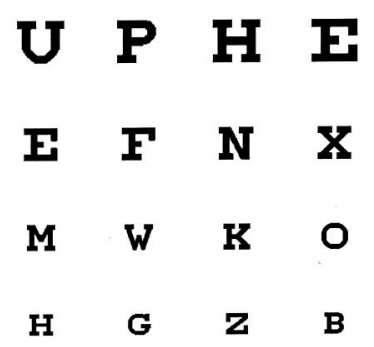

\section{Wave aberration}

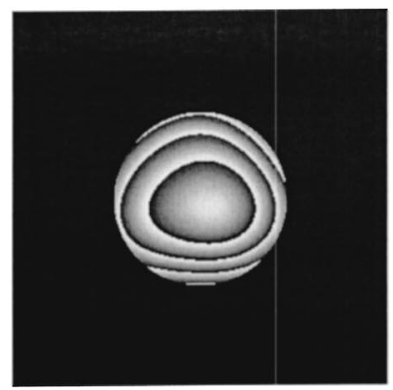

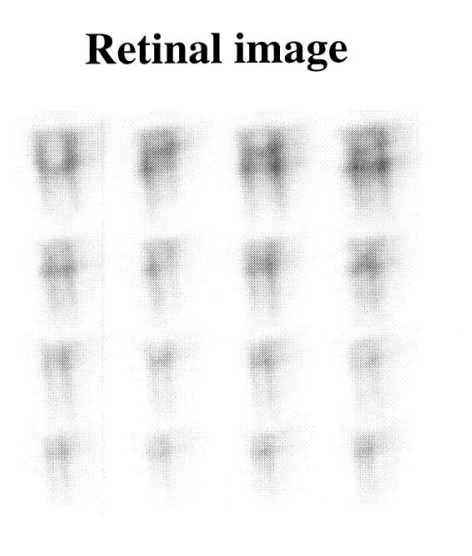

Recognition

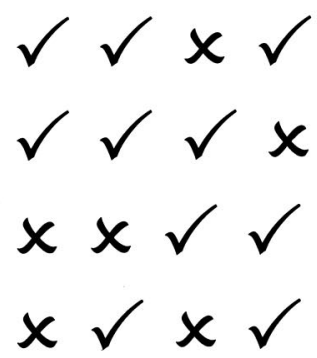

VA

0.6

0.8

1

1.2

\section{Alphabet \\ BCDEFGHKMNOP RUVWXZ}

Fig. 3. Illustration of the stages of the model. The input chart and the wave aberration are shown in the upper row. The retinal optical image, the result of the recognition, and the resulting visual acuity are shown the middle row $(\sqrt{ }$ and $\times$ mean correct or wrong answers, respectively). The reduced alphabet of 18 characters used in the recognition process is displayed at the bottom.

quently, these values were given less weight when the mean and standard deviation were computed. We applied standard values for all model input data (StilesCrawford apodization, distribution, parameters of Gabor filters, neural contrast threshold) except for the ocular aberration, where we used the Zernike coefficients corresponding to the experimentally measured optical aberrations of the subject's right eye. ${ }^{7}$

In the second example, aberration data from patients before and after LASIK surgery were used to compare the VA predicted by the model before and after surgery. Here a direct comparison with the model was even harder because VA was obtained in the ophthalmologic clinic, without measurement of the pupil size, and under best subjective refraction, which can be slightly different from that given by aberrometry. Nevertheless, the goal with these LASIK patients was not a further validation of the model but to show the potential effect of this kind of surgery on VA.

The third example corresponds to an optically ideal aberration-free eye, to illustrate the maximum predicted VA attainable by a complete compensation of optical aberrations, both monochromatic and polychromatic.

\section{RESULTS}

Figure 4 shows the results obtained in the first illustrative example, corresponding to subject RN. The predicted VA for different refractive states (defocus) is plotted for the monochromatic (solid curve) and polychromatic (dotted curve) cases. The astigmatism (-0.75 D) was left uncorrected. We followed the convention suggested by the Optical Society of America that positive defocus means myopic refractive state and negative defocus means hyperopic refractive state. Circles correspond to experimental VA measured in the optometry clinic as described in Section 3, with its corresponding error bars. The dashed curve is the predicted grating acuity, which tolerates much higher values of defocus, as expected. This suggests that in general it is not possible to extrapolate results from grating acuity to Snellen acuity.

From Fig. 4 we observe that the experimental VA peak is not at the circle of least confusion $(0 \mathrm{D})$, but at approximately $-0.75 \mathrm{D}$, close to the astigmatism foci. Once we pass the sturm interval, the subject is allowed to accommodate so that the VA is maintained at its maximum value. We did not test more negative defocus because it was clear from the high, saturated VA values that the 
subject was accommodating to compensate for the induced negative defocus. One interesting feature is that the predicted monochromatic VA shows several oscillations and is sharper than the polychromatic VA, mainly toward the myopic side. Introducing chromatic aberration smoothes the predicted values and eliminates some of the oscillations. In addition, the polychromatic VA is lower than the monochromatic one at the peak (minimum defocus), where the relative influence of the LCA is larger. The agreement between measured and predicted values for polychromatic light is highly satisfactory. There is a deviation at $1.0 \mathrm{D}$, which could be due to several factors, among them potential numerical artifacts caused by a large size of the point-spread function and the optotypes for these low values of VA, the failure of the OTF approximation in the Bayesian estimation model for large optical degradations, experimental artifacts (subject learning, etc.), and the use of standard values for several model parameters. Nevertheless, this is a promising result, which should be regarded only as a preliminary test of the model. A more rigorous psychophysical test, which is beyond the scope of this work, would require a more careful experimental design with a rigorous control of experimental conditions, real-time monitoring of pupil size, random presentation of optotypes to avoid learning the sequence by observers, and more subjects.

The second example illustrates how this model can be applied to practical problems, such as studying the change in predicted VA after refractive surgery. Figure 5 shows the predicted VA with the polychromatic version of the model for a patient before (solid curves) and after (dashed curves) LASIK refractive surgery and for two different pupil sizes (3.5 and $6.5 \mathrm{~mm})$. This example was taken from a previous study, ${ }^{10}$ where the wave aberration was measured in a group of patients before and after surgery. To avoid small values of VA, we assumed that in the preoperative case astigmatism $(1.75 \mathrm{D})$ was corrected. In contrast, residual astigmatism remaining after surgery $(0.5 \mathrm{D})$ is left uncorrected, assuming that the goal of sur-

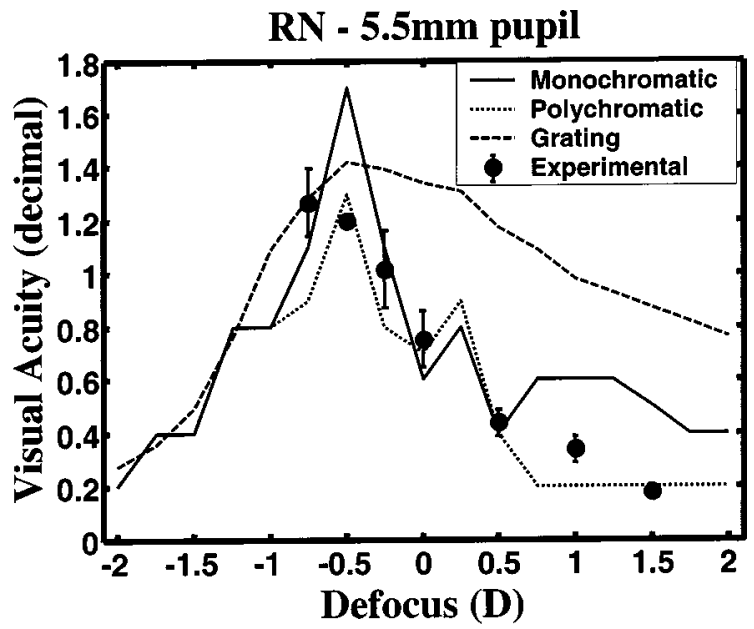

Fig. 4. Predicted visual acuity for different defocus values for subject RN in both monochromatic (solid curve) and polychromatic (dotted curve) light. Circles represent average experimental VA (polychromatic) from four runs of the experiment, with error bars corresponding to the standard deviation. Predicted grating detection acuity is also included (dashed curve).

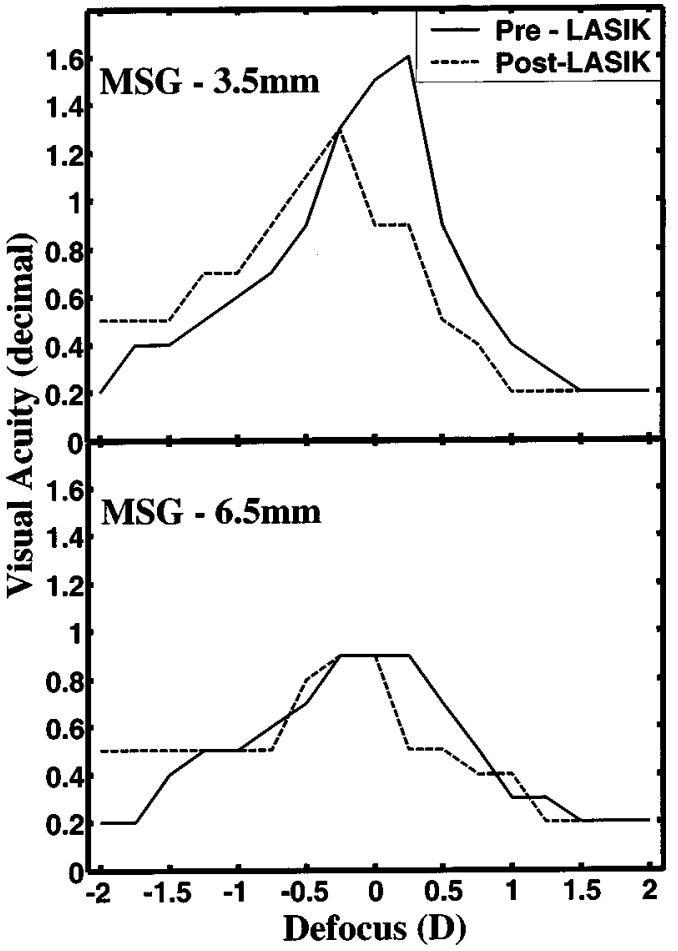

Fig. 5. Comparison of predicted VA in a patient before (solid curves) and after (dashed curves) LASIK refractive surgery, for 3.5- (upper panel) and 6.5- (lower panel) $\mathrm{mm}$ pupil diameters. The predicted VA suffers a slight decrease after surgery.

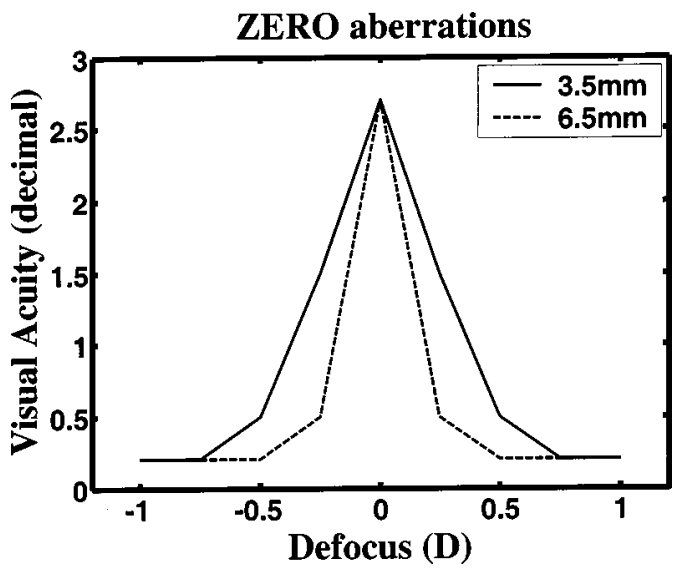

Fig. 6. Predicted VA for a perfect eye in which all aberrations have been corrected, for 3.5- (solid curve) and 6.5- (dotted curve) $\mathrm{mm}$ pupil diameters.

gery is to avoid the use of correcting lenses. As a result, in the preoperative case the VA peak value occurs at close to $0 \mathrm{D}$, whereas in the post-LASIK, case, it occurs closer to $-0.5 \mathrm{D}$. Residual astigmatism after surgery in addition to the increased higher-order aberrations (rms wave-front error is $0.77 \mu \mathrm{m}$ pre- and $1.0 \mu \mathrm{m}$ post-), causes both a shift and a reduction of the VA peak, especially for the 3.5-mm pupil. The effect of the increased aberrations with the $6.5-\mathrm{mm}$ pupil is a slight reduction in the depth of focus, as can be seen by the narrowing of the curve corresponding to the post-LASIK predictions relative to the curve before LASIK. For a 3.5-mm pupil (typical of day- 
light illumination conditions) the VA reaches peak values as high as 1.6 , whereas for a larger $6.5-\mathrm{mm}$ pupil, typical of nightlight conditions, the effect of aberrations is higher, reducing the peak value of the predicted VA to 0.9 . These results illustrate one potential application of the proposed method, and further work is needed to obtain stronger and more reliable conclusions.

In the last example we tested the VA of an ideal eye, setting all the monochromatic aberrations to 0 and scanning a range of $2 \mathrm{D}$ of defocus. Figure 6 plots the predicted values of VA of this ideal eye for two pupil sizes (3.5 and $6.5 \mathrm{~mm}$ ). The peak VA is 2.7 for both pupil sizes, much higher than any of the values in the previous examples. The main difference between pupil sizes is the slight decrease in depth of focus for the larger pupil, as expected. For both pupil sizes we observe that the resistance to defocus is lower than in the previous examples using real eyes with aberrations. Indeed, defocus is a degradation with contrast reversal and phase discontinuities that are not well modeled by the assumptions of our model, causing poor performance for even small amounts of defocus. When aberrations are present, this effect of defocus is diminished by the presence of other aberrations, and therefore the depth of focus is higher.

\section{DISCUSSION}

We have presented a Bayesian model of Snellen acuity that, as far as we know, is the first model that includes the three main stages of VA: (1) optical degradations and retinal sampling, (2) neural image representation and contrast thresholding, and (3) Bayesian pattern recognition. In this sense, a direct comparison with previous models is difficult, since they are limited either to predicting grating acuity ${ }^{16}$ or to reproducing the quality and visual appearance of the retinal image of optotype charts. ${ }^{17}$ Figure 4 showed that grating acuity may differ largely from Snellen acuity in the presence of aberrations. In fact, this figure shows that under in-focus conditions (minimum optical degradation) and with moderate aberrations (normal eye, 5.5-mm pupil) the discrepancy is small but that such discrepancy rapidly increases with defocus. Thus one of the most important conclusions is that Snellen acuity cannot be predicted solely from the MTF or from grating acuity, because these parameters ignore the influence of phase distortions (given by the phase transfer function), which can potentially be strong.

It is not easy to evaluate the accuracy of all the stages of the model. Although the theory of optical modeling is well established and optical measurements seem to be highly reliable, ${ }^{7}$ the uncertainty increases as we move toward neural processing. In this paper we have adopted schematic standard models. The basic underlying ideas are relatively well established ${ }^{21}$ even though there is still some controversy even about the actual existence of visual channels. Nevertheless, in the Bayesian model the use of visual channels tuned to specific frequencies and orientations appears to be a very convenient way to sample the optical degradation (the OTF) to constrain and simplify the estimation problem. This solution tolerates a high amount of degradation in the recognition stage. In fact, it is well known that standard pattern rec- ognition methods based on cross-correlation, phase-only filters, and the like, fail even with small amounts of defocus. We have attempted these standard methods, obtaining very poor values of VA even in best-corrected $(0 \mathrm{D}$ of defocus and astigmatism) normal eyes. On the other hand, results have demonstrated that predictions using the proposed multichannel scheme are in good agreement with experimental results except for low values of VA. The rest of the model also follows the standard paradigm, that an ideal observer will behave as a Bayesian "decision maker," 18 and the difference between the ideal and the real observer is introduced in terms of a given noise level or, as in our case, as a threshold.

One important assumption of the model is that the ideal shape of the optotypes has been previously learned by the subjects. Although one can argue that the subject is learning the optotypes from the retinal images, which are already affected by the degradation introduced by the optics, there are several drawbacks in considering a model in which the subject has learned a degraded version of the optotypes. First, the optical degradation is not constant and varies heavily with factors such as pupil size, accommodative status, or even externally induced defocus. Therefore the subject should learn a different version of the optotypes for each degradation and optotype scale, which seems less realistic than having a unique internal representation of the optotypes. Second, learning usually occurs in optimal conditions, with maximum contrast and a size large enough that the degradation introduced by the optics is negligible. On the other hand, it is also true that our model ignores the knowledge that human observers have about their own optics, which could be used to obtain an internal representation of the optotypes closer to the observed degraded ones and which would help to simplify the recognition problem.

Nevertheless, we have implemented a version of the model closer to the classical ideal-observer paradigm, ${ }^{18}$ in which the test optotypes and the stored alphabet are subject to the same degradation, given by a combination of the OTF and the NTF. After this, we add white noise to the degraded test image and proceed to a direct comparison with the stored degraded alphabet, using phase correlation (standard correlation gave poor performance). Figure 7 plots a comparison of this global channel model with our multichannel model, as well as the experimental VA measures, for subject RN with consideration of chromatic aberrations. We first notice that large error bars are affecting the global phase-correlation predictions. While running tests to adjust the noise power (it was finally set to $10^{-4}$ ), we noticed that the outcome of the phase-correlation method was highly variable depending on the noise realization, and therefore we decided to run the method using five different noise realizations and to show the mean and standard deviation on the graphs. This large variability is a serious disadvantage of this method, which otherwise does not give a better fit to the experimental data. We believe that phase correlation is unstable, so that small variations in the noise realization cause large variability on the outcome of the procedure. In addition, the noise level had to be empirically adjusted, whereas an important feature of the multichannel model proposed here is that we initially avoided any fitting of 


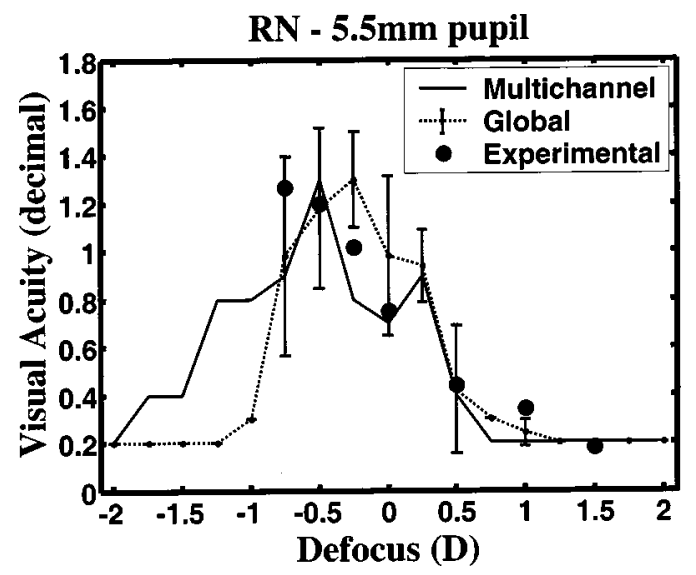

Fig. 7. Comparison of the VA predicted in polychromatic light by the multichannel model (solid curve) and by the global model (dotted curve). The circles show the average experimental VA. The error bars on the global model predictions correspond to the standard deviation in the predicted values VA from five different noise realizations.

free parameters. We propose the use of specific data measured on the subject in all the stages of the model when possible or the use of standard values when no specific data are available.

We have a monochromatic and a polychromatic version of the model. In the latter case, we adopted a rough approximation, looking for the simplest way to include the essential contribution of chromatic aberrations. ${ }^{34,35}$ Even the crude approximation of using three wavelengths and ignoring the TCA captures the strong influence of the chromatic aberrations, as can be checked in the comparison in Fig. 4.

The main objective of this work is to present a new Bayesian model for VA. The results presented here are illustrative. Nevertheless, we want to do further work to improve the chromatic model and study the relative influence of both LCA and TCA, extending the current monocular version to the binocular case, and testing the predictions of the model more rigorously.

\section{APPENDIX A}

Here we compute the parameters $\left(\hat{\mathbf{u}}_{i}^{j}, \hat{h}_{i}^{j}\right)$ that maximize the error function in relation (13), $E_{i}^{j}=\Sigma_{\mathbf{x}}\left[o_{i}(\mathbf{x})\right.$ $\left.-h_{i} c_{i}^{j}\left(\mathbf{x}-\mathbf{u}_{i}\right)\right]^{2}$. Before taking partial derivatives with respect to the parameters, we expand the square of the error function as follows:

$$
\begin{aligned}
E_{i}^{j}= & \sum_{\mathbf{x}}\left[o_{i}(\mathbf{x})\right]^{2}+\left(h_{i}\right)^{2} \sum_{\mathbf{x}}\left[c_{i}^{j}(\mathbf{x})\right]^{2} \\
& -2 h_{i} \sum_{\mathbf{x}} o_{i}(\mathbf{x}) c_{i}^{j}\left(\mathbf{x}-\mathbf{u}_{i}\right) .
\end{aligned}
$$

To minimize the error function in Eq. (A1) we take partial derivatives and equate to 0 :

$$
\frac{\partial E_{i}^{j}}{\partial h_{i}}=2 h_{i} K_{i}^{j}-2 \sum_{\mathbf{x}} o_{i}(\mathbf{x}) c_{i}^{j}\left(\mathbf{x}-\mathbf{u}_{i}\right)=0,
$$

$$
\frac{\partial E_{i}^{j}}{\partial \mathbf{u}_{i}}=2 h_{i} \sum_{\mathbf{x}} o_{i}(\mathbf{x}) \frac{\partial c_{i}^{j}\left(\mathbf{x}-\mathbf{u}_{i}\right)}{\partial \mathbf{u}_{i}}=0
$$

The condition in the second line is independent of $h_{i}$, and it is exactly the same condition that follows from maximizing the correlation $\operatorname{corr}_{i}^{j}\left(\mathbf{u}_{i}\right)=\Sigma_{\mathbf{x}} o_{i}(\mathbf{x}) c_{i}^{j}\left(\mathbf{x}-\mathbf{u}_{i}\right)$. Therefore, once we find the $\hat{\mathbf{u}}_{i}^{j}$ that maximizes the correlation, it follows from Eqs. (A2) that $\hat{h}_{i}^{j}=\operatorname{corr}_{i}^{j}\left(\hat{\mathbf{u}}_{i}^{j}\right) / K_{i}^{j}$.

\section{ACKNOWLEDGMENTS}

This research was partly supported by the Comisón Interministerial de Ciencia y Technología under grant DPI2002-04370-C02-02 and by the Centro del Laser Refractivo de Madrid. We also thank the two reviewers, whose comments helped to improve the quality of the manuscript.

Corresponding author Rafael Navarro can be reached by e-mail, r.navarro@io.csic.es.

\section{REFERENCES}

1. G. Smith, "Ocular defocus, spurious resolution and contrast reversal," Ophthalmic Physiol. Opt. 2, 398-404 (1982).

2. H. B. Peters, "The relationship between refractive error and visual acuity at three age levels," Am. J. Ophthalmol. 38, 194-199 (1961).

3. A. Bradley, T. Thomas, M. Kalaher, and M. Hoerres, "Effects of spherical and astigmatic defocus on acuity and contrast sensitivity: a comparison of three clinical charts," Optom. Vision Sci. 68, 418-426 (1991).

4. J. Liang, B. Grimm, S. Goelz, and J. Bille, "Objective measurement of wave aberrations of the human eye with the use of a Hartmann-Shack wave-front sensor," J. Opt. Soc. Am. A 11, 1949-1957 (1994).

5. R. Navarro and M. A. Losada, "Aberrations and relative efficiency of light pencils in the living human eye," Optom. Vision Sci. 74, 540-547 (1997).

6. J. C. He, S. Marcos, R. H. Webb, and S. A. Burns, "Measurement of the wave-front aberration of the eye by a fast psychophysical procedure," J. Opt. Soc. Am. A 15, 2449-2456 (1998).

7. E. Moreno-Barriuso and R. Navarro, "Laser ray tracing versus Hartmann-Shack sensor for measuring optical aberrations in the human eye," J. Opt. Soc. Am. A 17, 974-985 (2000).

8. P. Mierdel, H. E. Krinke, W. Wiegand, M. Kaemmerer, and T. Seiler, "Measuring device for determining monochromatic aberration of the human eye," Ophthalmologe 94, 441-445 (1997).

9. T. Seiler, M. Kaemmerer, P. Mierdel, and H. E. Krinke, "Ocular optical aberrations after photorefractive keratectomy for myopia and myopic astigmatism," Arch. Ophthalmol. 118, 17-21 (2000).

10. E. Moreno-Barriuso, J. Merayo-Lloves, S. Marcos, R. Navarro, L. Llorente, and S. Barbero, "Ocular aberrations before and after myopic corneal refractive surgery: LASIKinduced changes measured with laser ray tracing," Invest. Ophthalmol. Visual Sci. 42, 1396-1403 (2001).

11. W. Verdon, M. Bullimore, and R. K. Maloney, "Visual performance after photorefractive keratectomy," Arch. Ophthalmol. 114, 1465-1472 (1996).

12. J. T. Holladay, D. R. Dudeja, and J. Chang, "Functional vision and corneal changes after laser in situ keratomileusis determined by contrast sensitivity, glare testing and corneal topography," J. Cataract Refract. Surg. 25, 663-669 (1999).

13. R. A. Applegate, H. C. Howland, R. P. Sharp, A. J. Cottingham, and R. W. Yee, "Corneal aberrations and visual perfor- 
mance after radial keratotomy,” J. Refract. Surg. 14, 397407 (1998).

14. M. Giles, "Aberration tolerances for visual optical systems," J. Opt. Soc. Am. 67, 634-643 (1977).

15. M. Mrochen, M. Kaemmerer, and T. Seiler, "Wavefrontguided laser in situ keratomileusis: early results in three eyes," J. Refract. Surg. 16, 116-121 (2000).

16. J. E. Greivenkamp, J. Schwiegerling, J. M. Miller, and M. D. Mellinger, "Visual acuity modeling using optical raytracing of schematic eyes," Am. J. Ophthalmol. 120, 227-240 (1995).

17. A. Guirao, J. Porter, D. R. Williams, and I. G. Cox, "Calculated impact of higher-order monochromatic aberrations on retinal image quality in a population of human eyes," J. Opt. Soc. Am. A 19, 1-9 (2002).

18. W. S. Geisler, "Ideal-observer analysis of visual discrimination," in Frontiers of Visual Science (National Academy Press, Washington, D.C., 1987).

19. M. Born and E. Wolf, Principles of Optics, 6th ed. (Pergamon, Oxford, UK, 1993).

20. A. B. Watson, "Efficiency of a model human image code," J. Opt. Soc. Am. A 4, 2401-2417 (1987).

21. M. S. Landy and J. A. Movshon, Computational Models of Visual Processing (MIT Press, Cambridge, Mass., 1991).

22. F. W. G. Campbell and D. G. Green, "Optical and retinal factors affecting visual resolution,” J. Physiol. (London) 181, 576-593 (1965).

23. M. A. Losada, R. Navarro, and J. Santamaria, "Relative contributions of optical and neural limitations to human contrast sensitivity at different luminance levels," Vision Res. 33, 2321-2336 (1993).

24. J. Liang and D. R. Williams, "Aberrations and retinal image quality of the normal human eye," J. Opt. Soc. Am. A 14, 2873-2883 (1997).

25. A. Vargas, J. Campos, and R. Navarro, "Invariant pattern recognition against defocus based on subband decomposition of the filter," Opt. Commun. 185, 33-40 (2000).

26. J. Goodman, Introduction to Fourier Optics, 2nd ed. (McGraw-Hill, New York, 1996).

27. E. Peli, "Contrast in complex images," J. Opt. Soc. Am. A 7, 2032-2040 (1990).
28. H. H. Hopkins and M. J. Yzuel, "The computation of diffraction patterns in the presence of aberrations," Opt. Acta 17, 157-182 (1970).

29. L. N. Thibos, R. A. Applegate, J. T. Schwiegerling, and R. Webb, "Standards for reporting the optical aberrations of eyes," in Vision Science and Its Applications, V. Lakshminarayanan, ed., Vol. 35 of OSA Trends in Optics and Photonics Series (Optical Society of America, Washington, D.C., 2000), pp. 232-244.

30. R. A. Applegate and V. Lakshminarayanan, "Parametric representation of Stiles-Crawford functions: normal variation of peak location and directionality," J. Opt. Soc. Am. A 10, 1611-1623 (1993).

31. A. Van Meeteren, "Calculations on the optical modulation transfer function of the human eye for white light," Opt. Acta 21, 395-412 (1974).

32. G. Wyszecki and W. S. Stiles, Color Science: Concepts and Methods, Quantitative Data and Formulae (Wiley, New York, 1982).

33. R. Navarro, J. Santamaría, and J. Bescós, "Accommodationdependent model of the human eye with aspherics," J. Opt. Soc. Am. A 2, 1273-1281 (1985).

34. S. Marcos, S. A. Burns, E. Moreno-Barriuso, and R. Navarro, "A new approach to the study of ocular chromatic aberrations," Vision Res. 39, 4309-4323 (1999).

35. L. N. Thibos, A. Bradley, D. L. Still, X. Zhang, and P. A. Howarth, "Theory and measurement of ocular chromatic aberration," Vision Res. 30, 33-49 (1990).

36. O. Nestares, R. Navarro, J. Portilla, and A. Tabernero, "Efficient spatial-domain implementation of a multiscale image representation based on Gabor functions," J. Electron. Imaging 7, 166-173 (1998).

37. C. A. Curcio, K. R. Sloan, R. E. Kalina, and A. E. Hendrickson, "Human photoreceptor topography," J. Comp. Neurol. 292, 497-523 (1990).

38. D. R. Williams, "Visibility of interference fringes near the resolution limit," J. Opt. Soc. Am. A 2, 1087-1093 (1985).

39. P. Artal and R. Navarro, "Monochromatic modulation transfer function of the human eye for different pupil diameters: an analytical expression," J. Opt. Soc. Am. A 11, 246-249 (1994). 